\begin{tabular}{|ll||}
\hline Citation & $\begin{array}{l}\text { Xin Wang, Jan Swevers } \\
\text { Title } \\
\text { Offset-free Energy-optimal Model Predictive Control for Point- } \\
\text { to-point Motions with high positioning accuracy, page 60-65 }\end{array}$ \\
\hline Archived version & $\begin{array}{l}\text { Author manuscript: the content is identical to the content of the } \\
\text { published paper, but without the final typesetting by the publisher }\end{array}$ \\
\hline Published version & \\
\hline Journal homepage & $\begin{array}{l}\text { xin.wang@mech.kuleuven.be } \\
\text { your phone number + 32 (0)16 322222 }\end{array}$ \\
\hline Author contact & url in Lirias https://lirias.kuleuven.be/handle/123456789/481696 \\
\hline \hline
\end{tabular}




\title{
Offset-free Energy-optimal Model Predictive Control for Point-to-point Motions with High Positioning Accuracy
}

\author{
Xin Wang ${ }^{1}$ and Jan Swevers ${ }^{2}$
}

\begin{abstract}
This paper discusses Offset-free Energy-optimal Model Predictive Control (offset-free EOMPC) which is a MPC algorithm to realize time-constrained energy-optimal point-topoint motion control with high positioning accuracy for linear time-invariant (LTI) systems. The offset-free EOMPC approach is developed based on our previous research - Energy-optimal Model Predictive Control (EOMPC) - which aims at performing energy-optimal point-to-point motions within a given motion time. A drawback of the EOMPC method is that it cannot achieve high positioning accuracy in the presence of unmodelled disturbances or model-plant mismatch. In order to cope with this problem, a 'disturbance model' strategy is adopted: the system state is augmented with disturbance variables. Based on the 'disturbance model', the disturbances are estimated and the effect of which is cancelled. Numerical validation of the offsetfree EOMPC using a model of a linear motor with coulomb friction and cogging disturbances has been performed and the results show that time-constrained energy-optimal pointto-point motion with high positioning accuracy is achieved.
\end{abstract}

\section{INTRODUCTION}

Model predictive control (MPC) is an advanced control technique that determines the control action by solving online, at every sampling time, an open-loop optimal control problem, based on the current state of the system [1]-[3]. The optimization generates an input sequence for a specified time horizon. However, only the first input is applied to the system.

MPC algorithms became very popular in the eighties and were aimed to solve multivariable constrained control problems typical for the oil and chemical industries due to the limitation of the computation efficiency. Since the end of the nineties, the research on the development of fast MPC solution methods ('fast MPC' in short) has intensified to extend its application area to systems with fast sampling rates such as mechatronic or motion systems. Nowadays, an important application for this type of system is energy-optimal point-to-point motion control due to the growing social awareness to achieve an environmentally sustainable future. Our previous work EOMPC [4]-[5] has been developed for this type of applications. A main drawback of EOMPC is that a mechanisms is lacking to reduce or eliminate steadystate positioning errors caused by unmodelled disturbances or plant-model mismatch. This paper proposes an extension of EOMPC to cope with this problem.

In the MPC framework, offset-free control means that the controlled outputs are driven to their desired targets in steady

\footnotetext{
${ }^{1}$ Xin Wang is with Department of Mechanical Engineering, Division PMA, KU Leuven, Leuven, Belgium, Xin.Wang@mech.kuleuven.be

${ }^{2}$ Jan Swevers is with Department of Mechanical Engineering, Division PMA, KU Leuven, Leuven, Belgium, Jan.Swevers@mech.kuleuven.be
}

state, that is, the elimination of steady-state output errors is guaranteed. One way to achieve offset-free performance is suggested in [6]-[8]: the system state is augmented with an integrating disturbance. Inspired by this idea, Offset-free model predictive control methods [9]-[11] propose a general disturbance model that accommodates disturbances entering through the process input, state, or output. Based on the general disturbance model, the unmeasured disturbances are estimated and the effect of which can be removed by shifting the input to its steady-state target value so that offset-free control is achieved. These works only consider slow dynamic systems, sampling time of which is several seconds or even several minutes.

This paper discusses the EOMPC algorithm for mechatronic systems [5] in combination with the general disturbance model strategy proposed in [9], yielding the socalled offset-free EOMPC approach for linear-time invariant (LTI) systems. Offset-free EOMPC is able to perform time-constrained energy-optimal point-to-point motions with high positioning accuracy. Energy optimality is achieved by setting the object function of the optimization problem equal to the system's energy losses. The required motion time is guaranteed by minimizing the settling time of the system until the requested motion time is reached. The general disturbance model strategy is the key to achieve high positioning accuracy.

Outline of the paper: Section II and III briefly discuss the offset-free MPC approach [9] and EOMPC approach [5], respectively. Section IV explains the formulation of the developed offset-free EOMPC approach. Numerical validation of the developed method is shown in section V. In addition, a comparison with EOMPC and offset-free MPC is provided. Conclusions are drawn in the last section.

\section{OFFSET-FREE MODEL PREDICTIVE CONTROL}

For readers' convenience, this section summarizes two key steps of the offset-free MPC methods [9]: (i) construction of a general disturbance model that accommodates unmeasured disturbances entering through the process input, state, or output. (ii) calculation of the target input such that the effect of the disturbance is cancelled by shifting the control input to its target value.

\section{A. General Disturbance Model}

In this paper, we consider a discrete-time linear timeinvariant (LTI) system described by the following state-space 
model equation:

$$
\begin{aligned}
\mathbf{x}_{k+1} & =\mathbf{A} \mathbf{x}_{k}+\mathbf{B} u_{k}, \\
y_{k} & =\mathbf{C} \mathbf{x}_{k},
\end{aligned}
$$

where $y \in \mathbb{R}^{p}$ is the output, $u \in \mathbb{R}^{m}$ is the input, and $\mathbf{x} \in \mathbb{R}^{n}$ is the state of the system. It is assumed that $(\mathbf{A}, \mathbf{B})$ is stabilizable and $(\mathbf{C}, \mathbf{A})$ is detectable.

In order to achieve offset-free control, we augment the system state with a disturbance vector $\mathbf{d}_{k} \in \mathbb{R}^{n_{d}}$ and the resulting augmented system is defined in Eq. (2), where matrices $\mathbf{B}_{\mathbf{d}} \in \mathbb{R}^{n \times n_{d}}$ and $\mathbf{C}_{\mathbf{d}} \in \mathbb{R}^{p \times n_{d}}$ determine how the disturbances influence the system (either through the input, output or system state). The choice of the matrices is discussed below.

$$
\begin{aligned}
{\left[\begin{array}{l}
\mathbf{x}_{k+1} \\
\mathbf{d}_{k+1}
\end{array}\right] } & =\underbrace{\left[\begin{array}{cc}
\mathbf{A} & \mathbf{B}_{\mathbf{d}} \\
\mathbf{0} & \mathbf{I}
\end{array}\right]}_{\widetilde{\mathbf{A}}} \underbrace{\left[\begin{array}{c}
\mathbf{x}_{k} \\
\mathbf{d}_{k}
\end{array}\right]}_{\widetilde{\mathbf{x}}_{k}}+\underbrace{\left[\begin{array}{c}
\mathbf{B} \\
\mathbf{0}
\end{array}\right]}_{\widetilde{\mathbf{B}}} u_{k}+w_{k}, \\
y_{k} & =\underbrace{\left[\begin{array}{ll}
\mathbf{C} & \mathbf{C}_{\mathbf{d}}
\end{array}\right]}_{\widetilde{\mathbf{C}}}\left[\begin{array}{l}
\mathbf{x}_{k} \\
\mathbf{d}_{k}
\end{array}\right]+v_{k},
\end{aligned}
$$

in which $w_{k} \in \mathbb{R}^{n+n_{d}}$ and $v_{k} \in \mathbb{R}^{p}$ are noise vectors for the system state and output respectively. In matrix $\widetilde{\mathbf{A}}$, $\mathbf{I}$ is an identity matrix and it means that in absence of $w_{k}, d_{k+1}=d_{k}$ is assumed.

The estimate of the system state $\hat{\mathbf{x}}_{k \mid k}$ and the disturbance $\hat{d}_{k \mid k}$ at each sampling instance $k$ is formulated in Eq. (3)-(4) and follows the classical Luenberger approach [12]. It consists of two steps. First a 'prediction step' is performed, Eq.(3), yielding $\hat{\mathbf{x}}_{k \mid k-1}$ and $\hat{\mathbf{d}}_{k \mid k-1}$ based on the known information at time $k-1$. Eq. (4) is the second step, the 'correction step', and updates the prediction with a 'correction term', which is based on $y_{k}$, the measured output at time $k$.

1) 'prediction' step:

$$
\begin{aligned}
{\left[\begin{array}{c}
\hat{\mathbf{x}}_{k \mid k-1} \\
\hat{d}_{k \mid k-1}
\end{array}\right] } & =\widetilde{\mathbf{A}}\left[\begin{array}{l}
\hat{\mathbf{x}}_{k-1 \mid k-1} \\
\hat{d}_{k-1 \mid k-1}
\end{array}\right]+\widetilde{\mathbf{B}} u_{k-1}, \\
\hat{y}_{k} & =\widetilde{\mathbf{C}}\left[\begin{array}{l}
\hat{\mathbf{x}}_{k \mid k-1} \\
\hat{d}_{k \mid k-1}
\end{array}\right] .
\end{aligned}
$$

2) 'correction' step:

$$
\left[\begin{array}{c}
\hat{\mathbf{x}}_{k \mid k} \\
\hat{d}_{k \mid k}
\end{array}\right]=\left[\begin{array}{c}
\hat{\mathbf{x}}_{k \mid k-1} \\
\hat{d}_{k \mid k-1}
\end{array}\right]+\mathbf{L}\left(y_{k}-\hat{y}_{k}\right),
$$

where $\mathbf{L}$ is the gain matrix of the state estimator, which can be obtained using the pole placement approach [13] or the Kalman filter strategy [14].

\section{B. Target Calculation}

Offset-free MPC requires the controlled output $y$ to be equal to the reference output, $y=y_{r e f}$, in steady state. At each time step $l$, assuming that the estimate of the disturbance $\hat{d}_{l \mid l}$ is equal to the real disturbance $d_{l}, \hat{d}_{l \mid l}=d_{l}$, the steady-state condition is defined as follows:

$$
\left[\begin{array}{cc}
\mathbf{I}-\mathbf{A} & -\mathbf{B} \\
\mathbf{C} & \mathbf{0}
\end{array}\right]\left[\begin{array}{l}
\mathbf{x}_{r e f} \\
u_{r e f}
\end{array}\right]=\left[\begin{array}{c}
\mathbf{B}_{\mathbf{d}} \hat{d}_{l \mid l} \\
y_{r e f}-\mathbf{C}_{\mathbf{d}} \hat{d}_{l \mid l}
\end{array}\right]
$$

where $\mathbf{x}_{r e f}$ and $u_{r e f}$ are the target values for the state and the input in steady state respectively. Combining the MPC optimization problem with the steady-state condition Eq. (5) yields the offset-free MPC [9] optimization problem, formulated in Eq. (6), that determines the control signal over a specified time horizon $N_{\max }$.

$$
\begin{aligned}
& \min _{\substack{y_{0}, \ldots, y_{N_{\max }} \\
u_{0}, \ldots, u_{N_{\max }-1}}} \sum_{k=0}^{N_{\max }-1}\left\|u_{k}-u_{r e f}\right\|_{\mathbf{R}_{u}}^{2}+\left\|y_{k}-y_{r e f}\right\|_{\mathbf{Q}_{y}}^{2}
\end{aligned}
$$

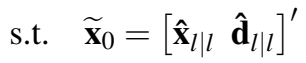

$$
\begin{aligned}
& \widetilde{\mathbf{x}}_{k+1}=\widetilde{\mathbf{A}} \widetilde{\mathbf{x}}_{k}+\widetilde{\mathbf{B}} u_{k}, \quad k=0,1, \cdots, N_{\max }-1 \\
& y_{k}=\widetilde{\mathbf{C}}_{k}, \quad k=0,1, \cdots, N_{\max } \\
& \underline{u} \leq E u_{k} \leq \bar{u}, \quad k=0,1, \cdots, N_{\max }-1 \\
& \underline{x} \leq F x_{k} \leq \bar{x}, \quad k=0,1, \cdots, N_{\max } \\
& {\left[\begin{array}{cc}
\mathbf{I}-\mathbf{A} & -\mathbf{B} \\
\mathbf{C} & \mathbf{0}
\end{array}\right]\left[\begin{array}{l}
\mathbf{x}_{r e f} \\
u_{r e f}
\end{array}\right]=\left[\begin{array}{c}
\mathbf{B}_{\mathbf{d}} \hat{d}_{l \mid l} \\
y_{r e f}-\mathbf{C}_{\mathbf{d}} \hat{d}_{l \mid l}
\end{array}\right],}
\end{aligned}
$$

where $\left(y_{0}, \ldots, y_{N_{\max }}, u_{0}, \ldots, u_{N_{\max }-1}\right)$ are the optimization variables over time horizon $N_{\max }$ and $\widetilde{\mathbf{x}}_{0}$ in Eq. (6b) is the current augmented state which contains the estimated system state $\hat{\mathbf{x}}_{l \mid l}$ and the estimated disturbance $\hat{\mathbf{d}}_{l \mid l}$ at time $l$. The control input sequence is optimized considering the Euclidean norm of the control effort and positioning error, weighted with semi-definite positivity matrices $\mathbf{R}_{u}$ and $\mathbf{Q}_{y}$ respectively, while respecting the current state $\widetilde{\mathbf{x}}_{0}$, disturbance model Eq. (6c-6d), system constraints Eq. (6e) and Eq. (6f) and steady-state condition Eq. (6g).

\section{ENERGY-OptimAL MPC}

In this section, the basic formulation of EOMPC is taken from our own previous work [5] and is repeated here for convenience. EOMPC is a MPC approach for linear timeinvariant (LTI) systems aiming at performing energy-optimal point-to-point motions within a required motion time $T[s]$. Energy optimality is achieved by setting the object function of the MPC optimization problem equal to the system's energy losses $E_{\text {loss }}$. In the EOMPC approach the settling time is defined as the number $N$ of discrete time sampling instants required for the system to be at rest at the desired set point. In order to guarantee the motion time, the settling time of the system is minimized until the requested motion time $T$ is reached. It is assumed that $T$ is integer multiple $K^{\star}$ of the sampling time $T_{s}, T=K^{\star} \times T_{s}$. Normally $N=K^{*}$ such that the motion time is guaranteed. If for some reason $T$ is too short considering the system constraints, EOMPC approach will automatically find the shortest possible motion time and realizes the motion in a time-optimal way.

The EOMPC optimization problem is formulated as a two-layer optimization problem [5]. The top layer is called 'Problem B' and determines the settling time. Finding the 
settling time involves solving a series of feasibility problems. This feasibility problem is the second layer and is called 'Problem A'. The optimal control sequence is the last feasible solution of 'Problem A'. Hence, at each time step $l$ the following two optimization problems are to be solved:

1) 'Problem A', denoted as $P_{A}\left(\hat{\mathbf{x}}_{l \mid l}, N\right)$, calculates the energy optimal control signal for a given settling time $N$ (which is obtained by solving 'Problem B') while respecting the system constraints as defined as follows.

$$
\begin{aligned}
V_{A}^{*}=\min _{\mathbf{u}} & E_{\text {loss }}(\mathbf{u}) \\
\text { s.t. } \quad \mathbf{x}_{0}=\hat{\mathbf{x}}_{l \mid l}, & \\
\mathbf{x}_{k+1}=\mathbf{A} \mathbf{x}_{k}+\mathbf{B} u_{k}, & k=0,1, \cdots, N_{\max }-1 \\
y_{k}=\mathbf{C} \mathbf{x}_{k}, & k=0,1, \cdots, N_{\max } \\
\underline{u} \leq E u_{k} \leq \bar{u}, & k=0,1, \cdots, N_{\max }-1 \\
\underline{x} \leq F x_{k} \leq \bar{x}, & k=0,1, \cdots, N_{\max } \\
y_{k}=y_{\text {ref }}, & k=N, \cdots, N+n-1 \\
u_{k}=0, & k=N, \cdots, N_{\max }-1
\end{aligned}
$$

In the object function (7a), energy losses $E_{\text {loss }}$ is minimized while respecting the current state $x_{0}$, the system model Eq. (7c) - (7d) and system constraints Eq.(7e) and Eq.(7f). In Eq.(7e) and Eq.(7f), the matrices $E$ and $F$ determine which input and state constraints are being considered respectively, e.g. if $E$ and $F$ are both defined as an identity matrix $I, \underline{u}$ and $\bar{u}$ in Eq.(7e) are the lower and upper bound of the system input and $\underline{x}$ and $\bar{x}$ in Eq.(7f) are the lower and upper bound of the system state. Eq. (7g-7h) are moving endpoint equality constraints and mean that no control input is imposed once the reference $y_{r e f}$ is reached hence the system is guaranteed to be at rest at $y_{\text {ref }}$ at time $N$. The outcome of problem $P_{A}\left(\hat{\mathbf{x}}_{l \mid l}, N\right)$ is that it is either feasible or not. Infeasibility of $P_{A}\left(\hat{\mathbf{x}}_{l \mid l}, N\right)$ means that the system can not be at rest at $y_{\text {ref }}$ within $N$ time steps while respecting system constraints Eq.(7e) and Eq.(7f). Therefore, an admissible set $\mathbb{X}(N)$ is defined:

$$
\mathbb{X}_{A}(N)=\left\{\hat{\mathbf{x}}_{l \mid l} \mid P_{A}\left(\hat{\mathbf{x}}_{l \mid l}, N\right) \text { is feasible }\right\},
$$

where $\mathbb{X}_{A}(N)$ is the set of system states from which $y_{\text {ref }}$ can be reached within $N$ time steps, while respecting all system constraints.

2) 'Problem B', denoted by $P_{B}\left(\hat{\mathbf{x}}_{l \mid l}\right)$, calculating the settling time $N$, is defined as follows:

$$
\begin{gathered}
V_{B}^{*}\left(\hat{\mathbf{x}}_{l \mid l}\right)=\min _{N \in \mathbb{N}} N \\
\text { s.t. } \quad \hat{\mathbf{x}}_{l \mid l} \in \mathbb{X}_{A}(N), \\
\max \left(K^{*}, N_{\text {min }}\right) \leq N \leq N_{\max },
\end{gathered}
$$

where $N$ is bounded by $N_{\max }$ and the maximum of $K^{*}$ and $N_{\min }$. To guarantee unconstrained solvability, $N_{\min }$ should be selected bigger than $n / m$ with $n$ the number of states and $m$ the number of inputs [15].
qpOASES, an open-source $\mathrm{C}++$ implementation of the online active set strategy [17]-[18], can be utilized to solve EOMPC problems efficiently.

\section{OFFSET-FREE ENERGY-OPTIMAL MPC}

Offset-free EOMPC is a control method to realize timeconstrained energy-optimal point-to-point motions with high positioning accuracy. It is developed by combining offsetfree MPC and EOMPC discussed in the previous two sections in one formulation. The offset-free EOMPC problem is formulated similar as EOMPC. The two-layer optimization problem of offset-free EOMPC consists of 'Problem C', $P_{C}\left(\hat{\mathbf{x}}_{l \mid l}, \hat{\mathbf{d}}_{l \mid l}, N\right)$, and 'Problem D', $P_{D}\left(\hat{\mathbf{x}}_{l \mid l}, \hat{\mathbf{d}}_{l \mid l}\right)$.

1) 'Problem $\mathrm{C}$ ', denoted by $P_{C}\left(\hat{\mathbf{x}}_{l \mid l}, \hat{\mathbf{d}}_{l \mid l}, N\right)$, calculating the offset-free time optimal control signal for a given $N$, which is obtained by solving 'Problem D', while respecting the system constraints, is formulated in the following :

$$
\begin{aligned}
& V_{C}^{*}=\min _{\mathbf{u}} E_{\text {loss }}(\mathbf{u}) \\
& \text { s.t. } \quad \widetilde{\mathbf{x}}_{0}=\left[\begin{array}{ll}
\hat{\mathbf{x}}_{l \mid l} & \hat{\mathbf{d}}_{l \mid l}
\end{array}\right]^{\prime}, \\
& \widetilde{\mathbf{x}}_{k+1}=\widetilde{\mathbf{A}} \widetilde{\mathbf{x}}_{k}+\widetilde{\mathbf{B}} u_{k}, \quad k=0,1, \cdots, N_{\max }-1 \\
& y_{k}=\widetilde{\mathbf{C}} \widetilde{\mathbf{x}}_{k}, \quad k=0,1, \cdots, N_{\max } \\
& \underline{u} \leq E u_{k} \leq \bar{u}, \quad k=0,1, \cdots, N_{\max }-1 \\
& \underline{x} \leq F x_{k} \leq \bar{x}, \quad k=0,1, \cdots, N_{\max } \\
& y_{k}=y_{r e f}, \quad k=N, \cdots, N+n-1 \\
& u_{k}=u_{\text {ref }}, \quad k=N, \cdots, N_{\max }-1 \\
& {\left[\begin{array}{cc}
\mathbf{I}-\mathbf{A} & -\mathbf{B} \\
\mathbf{C} & \mathbf{0}
\end{array}\right]\left[\begin{array}{l}
\mathbf{x}_{r e f} \\
u_{r e f}
\end{array}\right]=\left[\begin{array}{c}
\mathbf{B}_{\mathbf{d}} \hat{d}_{l \mid l} \\
y_{r e f}-\mathbf{C}_{\mathbf{d}} \hat{d}_{l \mid l}
\end{array}\right],}
\end{aligned}
$$

In offset-free EOMPC, system's energy losses $E_{\text {loss }}$ is minimized with respect to the current augmented state $\widetilde{\mathbf{x}}_{0}$, the disturbance model Eq. (10c-10d) and the system constraints Eq. (10e) and Eq. (10f). Given the disturbance estimate $\hat{\mathbf{d}}_{l \mid l}$, the target input $u_{r e f}$ calculated in Eq. (10i) is a none zero value. This means that for the moving endpoint equality constraints, $u_{k}$ in Eq. (10h) has to be equal to $u_{r e f}$ such that the system is guaranteed to be at rest at $y_{\text {ref }}$ at time $N$ as shown in Eq. (10g). Similar as EOMPC, problem 'C' defines an admissible set,

$$
\mathbb{X}_{C}(N)=\left\{\hat{\mathbf{x}}_{l \mid l} \mid P_{C}\left(\hat{\mathbf{x}}_{l \mid l}, \hat{\mathbf{d}}_{l \mid l}, N\right) \text { is feasible }\right\} .
$$

2) 'Problem D', denoted by $P_{D}\left(\hat{\mathbf{x}}_{l \mid l}, \hat{\mathbf{d}}_{l \mid l}\right)$, calculating the settling time $N$, is defined as follows:

$$
\begin{gathered}
V_{D}^{*}\left(\hat{\mathbf{x}}_{l \mid l}, \hat{\mathbf{d}}_{l \mid l}\right)=\min _{N \in \mathbb{N}} N \\
\text { s.t. } \quad \hat{\mathbf{x}}_{l \mid l} \in \mathbb{X}_{C}(N), \\
\max \left(K^{*}, N_{\min }\right) \leq N \leq N_{\max } .
\end{gathered}
$$

qpOASES is also utilized to solve the offset-free EOMPC optimization problems. 
Remark that with the offset-free EOMPC, positioning at $y_{r e f}$ is imposed using equality constraints Eq. (10g)-(10h). The offset-free MPC realizes positioning through the object function Eq. (6a) and an appropriate selection of $\mathbf{Q}_{y}$.

\section{NUMERICAL VALIDATION}

This section discusses the numerical validation of the developed offset-free EOMPC algorithm using a model of a linear motor test setup in our lab. Details of the linear motor are described in the following subsection.

\section{A. Considered Test Setup}

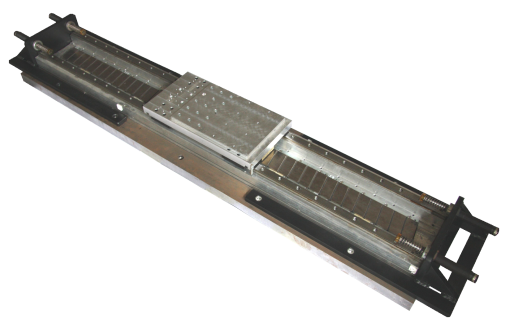

Fig. 1. The linear motor test setup

The considered test setup is a current-controlled permanent-magnet linear motor shown in Fig. 1. The control input to the system is motor current $[A]$, and the output is the position of the carriage $[m]$. Gaussian measurement noise with a standard distribution of $0.5 \mu \mathrm{m}$ is added to the carriage position. A second order discrete-time state-space model of the system as shown in Eq. (1) is identified, where dynamic matrices $\mathbf{A}=\left[\begin{array}{cc}1.8311 & -0.8311 \\ 1 & 0\end{array}\right], \mathbf{B}=\left[\begin{array}{ll}0.0156 & 0\end{array}\right]^{\prime}, \mathbf{C}=$ $\left[\begin{array}{ll}0.0144 & 0.0101\end{array}\right]$ with the sampling frequency $f_{s}=100 \mathrm{~Hz}$. The system poles are: one at $z=1$, corresponding to an integrator and the other one at $z=0.8311$ resulting from viscous friction. This model is a discrete-time equivalent of a mass-damper model actuated by a force input, which is proportional to the motor current.

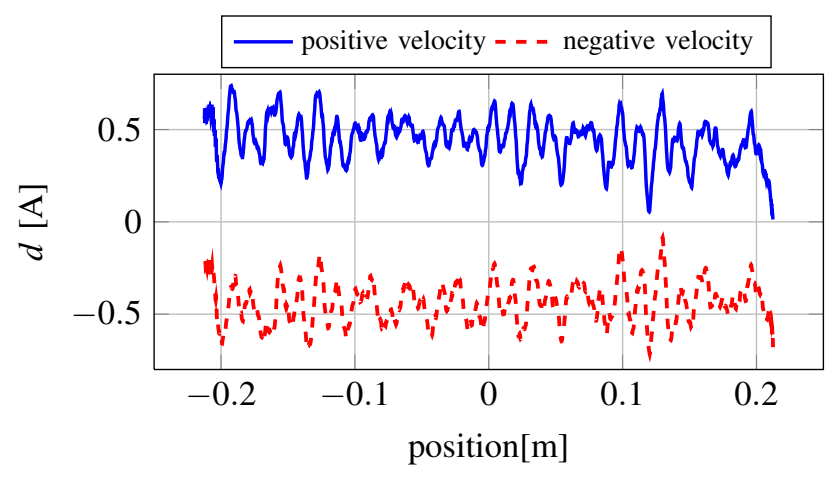

Fig. 2. Coulomb friction and cogging of the linear motor

The main disturbances in this test setup are Coulomb friction $d_{\text {cou }}[A]$ and cogging $d_{\operatorname{cog}}[A]$ and we define $d=$ $d_{\text {cou }}+d_{\text {cog }}$. Remark that the disturbance $d$ is expressed in $[A]$ because it is an input disturbance. Coulomb friction is a constant which depends on the sign of the velocity. Cogging, which is independent of motion velocity, is more or less a periodic function of the position and the period corresponds to the distance between the magnetic poles of the linear motor. [19] discusses the identification of the disturbance $d$ of this linear motor setup, the result of which is shown in Fig. 2. The solid and the dashed line represent disturbance $d$ for positive and negative velocity respectively.

Since the disturbance $d$ is an input disturbance, the disturbance matrices in the system model Eq. (2) are defined as $\mathbf{B}_{\mathbf{d}}=\mathbf{B}$ and $\mathbf{C}_{\mathbf{d}}=0$, yielding that the disturbance is added directly to the input. Hence, the augmented system matrices are defined as follows:

$$
\begin{aligned}
\widetilde{\mathbf{A}} & =\left[\begin{array}{ccc}
1.8311 & -0.8311 & 0.0156 \\
1 & 0 & 0 \\
0 & 0 & 1
\end{array}\right], \\
\widetilde{\mathbf{B}} & =\left[\begin{array}{lll}
0.0156 & 0 & 0
\end{array}\right]^{\prime}, \\
\widetilde{\mathbf{C}} & =\left[\begin{array}{lll}
0.0144 & 0.0101 & 0
\end{array}\right] .
\end{aligned}
$$

\section{B. Formulation of the Optimization Problem}

Energy losses of the linear motor, $E_{\text {loss }}$, are copper losses which are proportional to the square of the motor current $u$ [A] as shown in Eq. (14),

$$
E_{\text {loss }}=T_{s} \cdot \sum_{k=0}^{N_{\max }-1} c \cdot u_{k}^{2},
$$

where $c$ is a constant depending on the ohmic resistance of the motor. $T_{s}$ is the sampling time and $N_{\max }$ is length of the prediction horizon. Due to the limitations of the system, position and motor current constraints, $\pm 0.25 \mathrm{~m}$ and $\pm 3 \mathrm{~A}$ respectively, are taken into account.

$P_{C}\left(\hat{\mathbf{x}}_{l \mid l}, \hat{\mathbf{d}}_{l \mid l}, N\right)$ of the offset-free EOMPC optimization problem is hence defined as following:

$$
\begin{aligned}
& \min _{\mathbf{u}} \mathbf{u}^{\prime} \mathbf{R u} \\
& \text { s.t. } \quad \widetilde{\mathbf{x}}_{0}=\left[\begin{array}{ll}
\hat{\mathbf{x}}_{l \mid l} & \hat{\mathbf{d}}_{l \mid l}
\end{array}\right]^{\prime}, \\
& \widetilde{\mathbf{x}}_{k+1}=\widetilde{\mathbf{A}} \widetilde{\mathbf{x}}_{k}+\widetilde{\mathbf{B}} u_{k}, \quad k=0,1, \cdots, N_{\max }-1 \\
& y_{k}=\widetilde{\mathbf{C}} \widetilde{\mathbf{x}}_{k}, \quad k=0,1, \cdots, N_{\max } \\
& u_{\text {min }}, y_{\text {min }} \leq u_{k}, y_{k} \leq u_{\max }, y_{\max } \\
& y_{k}=y_{r e f}, \quad k=N, \cdots, N+n-1 \\
& u_{k}=u_{\text {ref }}, \quad k=N, \cdots, N_{\max }-1 \\
& {\left[\begin{array}{cc}
\mathbf{I}-\mathbf{A} & -\mathbf{B} \\
\mathbf{C} & \mathbf{0}
\end{array}\right]\left[\begin{array}{l}
\mathbf{x}_{r e f} \\
u_{r e f}
\end{array}\right]=\left[\begin{array}{c}
\mathbf{B} \hat{d}_{l \mid l} \\
y_{r e f}
\end{array}\right]}
\end{aligned}
$$

where $\widetilde{\mathbf{A}}, \widetilde{\mathbf{B}}, \widetilde{\mathbf{C}}$ are defined in Eq. (13). $\mathbf{R}$ in Eq. (15a) is equal to $c_{t} \times I$ with $I$ the identity matrix and $c_{t}=c \times T_{s}$, such that the cost function Eq. (15a) corresponds to $E_{l o s s}$ in Eq. (14). Remark that the value of $c_{t}$ does not influence the optimal solution and hence can be omitted. This optimization problem is a convex QP because the dynamics of the system and the constraints are linear. The on-line active set method qpOASES can be utilized to solve this optimization problem. 


\section{Simulation Results}

The comparison of the offset-free EOMPC, EOMPC and offset-free MPC are performed using the discussed test setup and disturbance model and the control scheme is shown in Figure 3. Measurement noise, with an rms value of $0.5 \mu \mathrm{m}$, is taken into account.

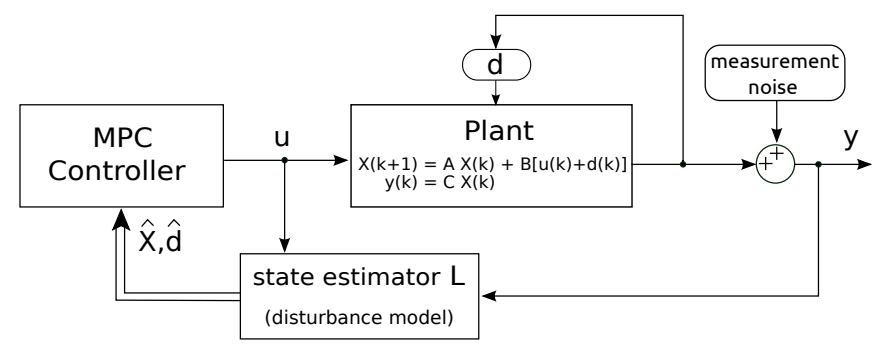

Fig. 3. Control scheme of the numerical validation using MPC controller (offset-free EOMPC, EOMPC or offset-free MPC)

The following case is considered: the system is initially at rest at position $0 \mathrm{~m}$, and a desired setpoint $y_{\text {ref }}=0.2 \mathrm{~m}$ is requested at time $t=0.4 \mathrm{~s}$. The required motion time $T=$ $0.6 \mathrm{~s}$, hence $K^{*}=T / T_{s}=60$. The prediction horizon $N_{\max }=$ $80>K^{*}, N_{\min }=6$ and the sampling rate $f_{s}=100 \mathrm{~Hz}$. The state estimator gain $L=\left[\begin{array}{llll}76.75 & 47.40 & 541.07\end{array}\right]^{\prime}$ is obtained using pole placement [13], yielding an estimator bandwidth of $13 \mathrm{~Hz}$, which corresponds for the considered case to a good trade-off between accuracy of the estimation and noise sensitivity.

Figure 4 - 6 show the simulation results. When the desired setpoint $y_{\text {ref }}=0.2 \mathrm{~m}$ is requested at $t=0.4 \mathrm{~s}$ as shown in Fig. 4(b) (dotted line), the requested motion time $T$ shown in Fig. 4(a) jumps to the given value $0.6 \mathrm{~s}$ and after that gradually decreases until it reaches zero at time $t=1 \mathrm{~s}$, at which time the position of the system using offset-free EOMPC arrives at the requested setpoint as shown in Fig. 4(b) (solid line) with high positioning accuracy as illustrated by its output error shown in Fig. 4(d). Using offset-free MPC, the system arrives at $y_{\text {ref }}$ with high positioning accuracy as well (dashed line), but the motion time $T$ can only be achieved approximately by tuning the weights $\mathbf{R}_{u}$ and $\mathbf{Q}_{y}$. In this case, $\mathbf{R}_{u}=I$ and $\mathbf{Q}_{y}=$ $1000 \times I$. However, the position of the system using EOMPC ( dash-dot line) has a large steady-state error due to the fact that the effect of the disturbance $d$ is not compensated.

Fig. 4 (c) and (d) show the system inputs and output errors respectively for these three methods. All the current inputs satisfy the constraints $\pm 3 \mathrm{~A}$. Only the current input using offset-free MPC (dashed line) reaches the maximum value 3A since it is not determined by minimizing the system's energy losses, which are proportional to square of the current. The output error using offset-free EOMPC is approximately $0.1 \mathrm{~mm}$ at time $t=1 \mathrm{~s}$, at which time the system is supposed to arrive at the setpoint. After that, the output error keeps decreasing and reaches its lowest value within $0.15 \mathrm{~s}$. The RMS of the remaining error is $0.5 \mu \mathrm{m}$ which corresponds to the measurement noise level. The output errors using offsetfree MPC and EOMPC are approximately $1 \mathrm{~mm}$ at time $t=$ (a)

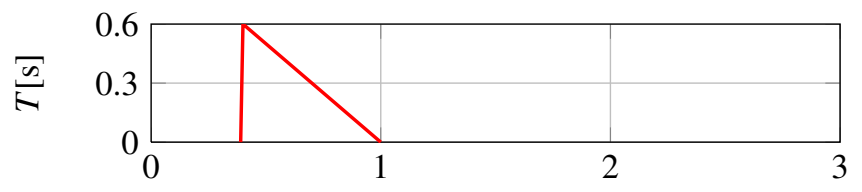

(b)

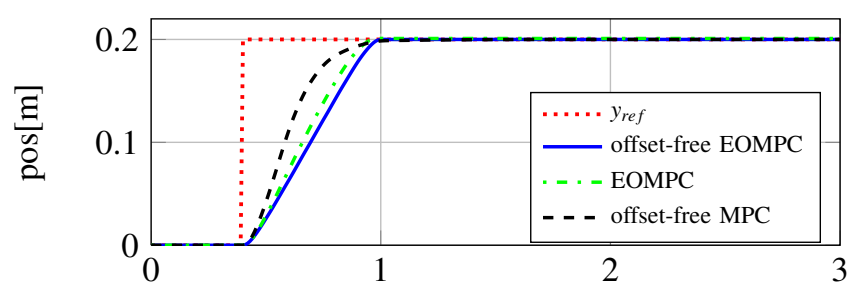

(c)

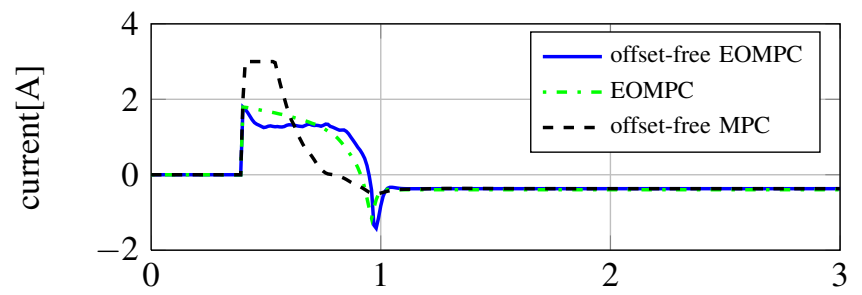

(d)

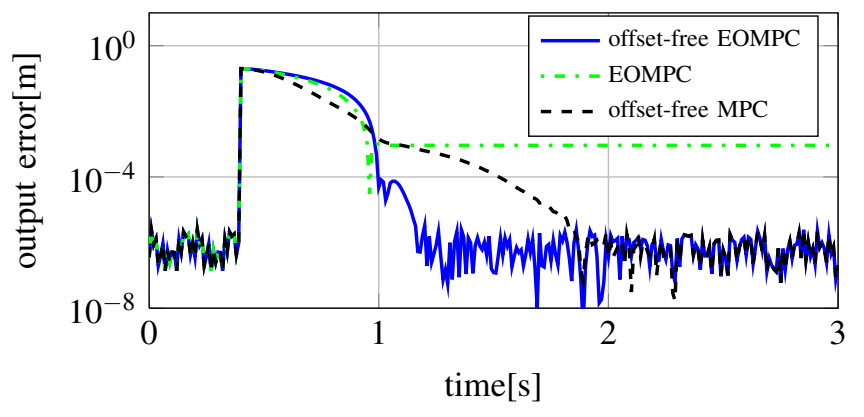

Fig. 4. Simulation results: (a) motion time, (b) carriage positions, (c) currents, (d) output error using offset-free EOMPC (solid), EOMPC (dashed) and offset-free MPC (dash-dot)

1s. The output error using EOMPC remains at this level, but offset-free MPC is able to further decrease the output error to an RMS value of $0.5 \mu \mathrm{m}$ within $1 \mathrm{~s}$, which is considerably slower than offset-free EOMPC. These results show that the offset-free EOMPC and offset-free MPC achieve the same positioning accuracy, but offset-free EOMPC is faster due to the fact that positioning is imposed directly using equality constraints Eq. (10g)-(10h).

The estimates of the disturbance using offset-free EOMPC (solid line) and offset-free MPC (dashed line) shown in Fig. 5 are almost same since they are obtained using the same state estimator gain $L$. Peaks of the disturbance $d$ are not estimated accurately because of the low bandwidth of the state estimator, but both estimated disturbances corresponds well to the average value of the disturbance $d$. Details of the estimated disturbances when the system approaching $y_{\text {ref }}$ are shown in Fig 6. Both estimates of the disturbance at $y_{r e f}$ are 


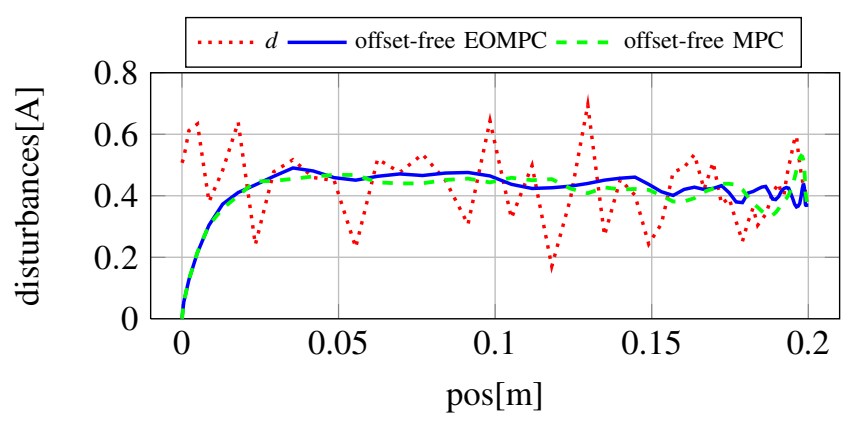

Fig. 5. Estimated disturbances using offset-free EOMPC (solid) and offsetfree MPC (dash dot)

equal to the disturbance $d$ at $y_{r e f}$, which is $d(0.2 \mathrm{~m})=0.369 \mathrm{~A}$.

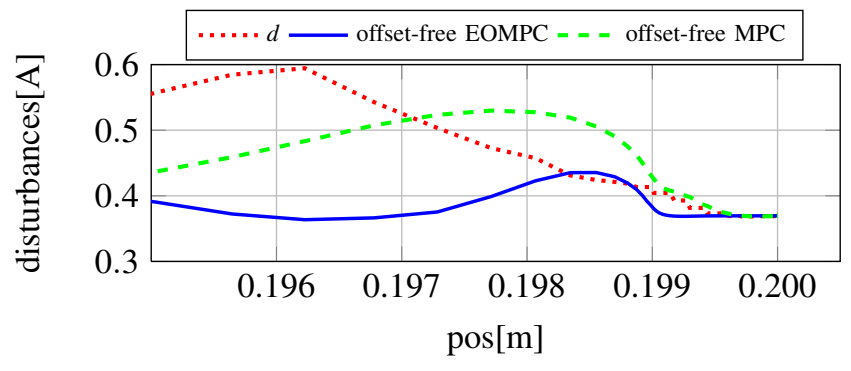

Fig. 6. Details of the estimated disturbances at $y_{\text {ref }}=0.2 \mathrm{~m}$

Based on above simulation results, the performances of the system using these three control methods are summarized in the table I:

TABLE I

NUMERICAL VALIDATION RESULTS

\begin{tabular}{|c|c|c|c|}
\hline Methods & $\frac{E_{\text {loss }}}{T_{s} \cdot c}$ & $R M S$ of output error & motion time \\
\hline offset-free EOMPC & 122.52 & $1 \mu \mathrm{m}$ & guaranteed \\
\hline EOMPC & 139.04 & $1 \mathrm{~mm}$ & guaranteed \\
\hline offset-free MPC & 200.81 & $1 \mu \mathrm{m}$ & approximately \\
\hline
\end{tabular}

According to the table, the energy losses of offset-free EOMPC is comparable to that of EOMPC and is $44.49 \%$ less than that of offset-free MPC. The positioning accuracy of offset-free EOMPC and offset-free MPC are equally good and $0.5 \mu \mathrm{m}$ is the best positioning accuracy that can be obtained due to the measurement noise. In short, offsetfree EOMPC achieves time-constrained point-to-point motion with the best positioning accuracy while guaranteeing energy optimality.

\section{CONCLUSIONS}

This paper presents offset-free energy-optimal MPC which aims at performing time-constrained energy-optimal pointto-point motions with high positioning accuracy. This approach is validated and compared with other two methods:
EOMPC and offset-free MPC, using a realistic model of a linear motor setup with Coulomb friction and cogging disturbances. The simulation results show that the offset-free EOMPC yields the best performance: energy-optimal motion with required motion time and accuracy positioning.

\section{ACKNOWLEDGMENT}

This work benefits from KU Leuven-BOF PFV/10/002 Center-of- Excellence Optimization in Engineering (OPTEC), the Belgian Programme on Interuniversity Attraction Poles, initiated by the Belgian Federal Science Policy Office (DYSCO) and KU Leuven's Concerted Research Action GOA/10/11.

\section{REFERENCES}

[1] M. Morari and J. Lee, Model predictive control: past, present and future, vol. 23, Computers and Chemical Engineering, 1999, pp. 667682.

[2] D. Mayne and J. Rawlings and C. Rao and P. Scokaert, Constrained model predictive control: Stability and optimality, vol. 36, Automatica, 2000, pp. 789-814.

[3] Jay H. Lee, Model predictive control: Review of the three decades of development, vol. 9, no. 3, International journal of control, autonation and systems, 2011, pp. 415-424.

[4] Xin Wang and Julian Stoev and Gregory Pinte and Jan Swevers, Energy Optimal Point-to-point Motion Using Model Predictive Control, 5th annual Dynamic Systems and Control Conference (DSCC), vol. 2, October 17, 2012, pp. 267-273.

[5] X. Wang, J. Stoev, G. Pinte and J. Swevers. Classical and modern methods for time-constrained energy optimal motion - Application to a badminton robot, Mechatronics, vol. 23, Issue 6, September 2013, pp. 669-676.

[6] E.J. Davison, and H.W. Smith, Pole assignment in linear timeinvariant multivariable systems with constant disturbances, vol. 7, no. 4, Automatica, 1971, pp. 489-498.

[7] E.J. Davison, and H.W. Smith, A note on the design of industrial regulators: Integral feedback and feedforward controllers, vol. 10, no. 3, Automatica, 1974, pp. 329-332.

[8] L. Qiu, and E.J. Davison, Performance limitations of non-minimum phase systems in the servomechanism problem, vol. 29, no. 2, Automatica, 1993, pp. 337-349.

[9] G. Pannocchia1, and J.B. Rawlings, Disturbance models for offsetfree model-predictive control, vol. 49, no. 2, AIChE Journal, 2003, pp. 426-437.

[10] M.R. Rajamani, and J.B. Rawlings, Achieving state estimation equivalence for misassigned disturbances in offset-free model predictive control, vol 55, no. 2, AIChE Journal, 2009, pp. 396-407.

[11] K.R. Muske, and T.A. Badgwell, Disturbance modeling for offsetfree linear model predictive control, vol. 12, no. 5, Journal of Process Control, 2002, pp. 617-632.

[12] David G. Luenberger, An introduction to observers, vol. 16, no. 6, IEEE transactions on Automatic control, 1971, pp. 596-602.

[13] G.F. Franklin, and D.J. Powell, and A. Emami Naeini, Feedback control of dynamic systems, Prentice Hall, 2001

[14] G. Welch, and G. Bishop, An introduction to the Kalman filter, 2004

[15] Kailath, T., Linear systems, Prentice Hall, 1980.

[16] Hans Joachim Ferreau et al., qpOASES Users Manual, March 2014.

[17] Ferreau,H.J., and Bock, H.G., and Diehl, M., An online active set strategy to overcome the limitations of explicit MPC, vol. 18, no. 8, International Journal of Robust and Nonlinear Control, 2008, pp. 816830.

[18] H.J. Ferreau, An Online Active Set Strategy for Fast Solution of Parametric Quadratic Programs with Applications to Predictive Engine Control, 2006.

[19] P. Janssens, and G. Pipeleers, and J. Swevers, Initialization of ILC based on a previously learned trajectory, American Control Conference, 2012, pp. 610-614.

[20] T. Kailath, Linear systems, Prentice Hall, 1980. 